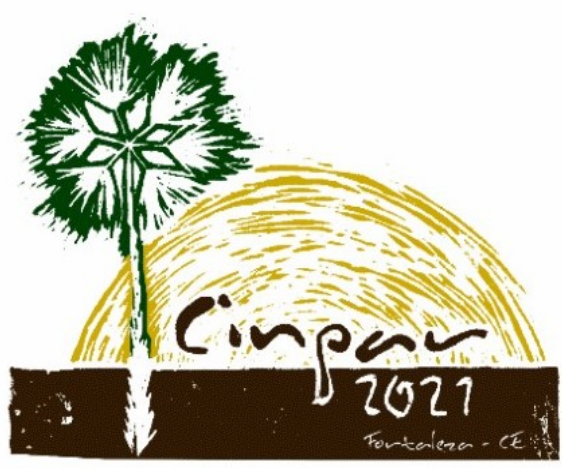

XVII Congresso Internacional sobre Patologia e Reabilitação das Construções

XVII Congreso Internacional sobre Patología y Rehabilitación de las Construcciones

XVII International Conference on Pathology and Constructions Rehabilitation

FORTALEZA (Brasil), 3 a 5 de junho de 2021 https://doi.org/10.4322/CINPAR.2021.079

\title{
Sistema computacional para identificar níveis de degradação em barras de aço
}

\section{Computer system to identify degradation levels in steel bars}

\author{
Alessandra I. P. dos SANTOS ${ }^{1}$, Eduarda S. GAVIÃO ${ }^{2}$, Kelly CHAPLA ${ }^{3}$, Carlos E. T. BALESTRA ${ }^{4}$, Fabio A. \\ SPANHOL ${ }^{5}$, Jefferson G. MARTINS ${ }^{6}$

\footnotetext{
${ }^{1}$ Universidade Tecnológica Federal do Paraná (UTFPR), Toledo, Brasil, aleiolandap@hotmail.com ${ }^{2}$ Universidade Tecnológica Federal do Paraná (UTFPR), Toledo, Brasil, eduardagaviao@hotmail.com ${ }^{3}$ Universidade Tecnológica Federal do Paraná (UTFPR), Toledo, Brasil, kelly_chapla@hotmail.com ${ }^{4}$ Universidade Tecnológica Federal do Paraná (UTFPR), Toledo, Brasil, carlosbalestra@utfpr.edu.br

${ }^{5}$ Universidade Tecnológica Federal do Paraná (UTFPR), Toledo, Brasil, faspanhol@utfpr.edu.br

${ }^{6}$ Universidade Tecnológica Federal do Paraná (UTFPR), Toledo, Brasil, martins@utfpr.edu.br
}

\begin{abstract}
Reinforcement corrosion in concrete structures is one of the most important pathological manifestations that leads to degradation and structural damage of buildings, while represents risks to people lives. Analysis and identification of degradation levels of these structures is characterized as a repetitive, monotonous and time-consuming process. In this scenario, computer systems based on computer vision have been applied to similar problems to replace or assist human specialists. This paper presents a proposal to classify different degradation levels in steel bars through computer vision. A widely known set of tools in the machine learning area was used and validated the proposal. The best results achieved recognition rates of $75.6 \%$ by using LPQ descriptor and SVM machine learning algorithm.
\end{abstract}

Keywords: Construction. Corrosion. Textural descriptors. Public security. Automation.

Resumo: A corrosão das armaduras em estruturas de concreto é uma das principais manifestações patológicas que leva a sua degradação e ao comprometimento estrutural das edificações, bem como impõe riscos a vidas humanas. A análise e identificação dos graus de degradação destas estruturas se caracteriza como um processo repetitivo, monótono e demorado. Diante deste cenário, sistemas computacionais que trabalhem com visão computacional têm sido aplicados a problemas semelhantes a este para substituir ou auxiliar especialistas humanos. Este artigo apresenta uma proposta para classificar diferentes graus de degradação em barras de aço por meio de visão computacional. Foram empregados ferramentais amplamente conhecidos na área de aprendizagem de máquina, cujos resultados validaram a proposta. Os melhores resultados alcançaram taxas de acerto de $75,6 \%$ usando o descritor LPQ e algoritmo de aprendizagem de máquinas SVM.

Palavras-chave: Construção civil. Corrosão. Descritores texturais. Segurança pública. Automação. 


\section{Introdução}

Identificada como um dos principais problemas relacionados à degradação de estruturas de concreto armado no mundo, a corrosão das armaduras pode demandar até $50 \%$ dos recursos investidos em construção civil para a reabilitação de estruturas (MEHTA et al., 2008). Segundo Cunha et al. (2013), tal problema consome uma parcela significativa do produto interno bruto de uma nação industrializada.

Além de financeiras, as consequências mais graves são possíveis danos corporais e até perdas de vidas humanas. Possíveis colapsos estruturais merecem atenção. Nos últimos anos têm sido recorrentes os casos de colapsos de estruturas devido à corrosão de armaduras. Dentre vários casos com repercussão na mídia nacional, pode-se citar o colapso parcial de um viaduto na cidade de Brasília em 2018 (VIADUTO, 2018) e a interdição do acesso à Rodovia Presidente Dutra, na cidade de São Paulo em 2019 (PREFEITURA, 2019).

A corrosão envolve reações de oxidação e de redução (redox) que convertem o metal ou componente metálico em óxidos ou hidróxidos expansivos (SILVA et al., 2015). A corrosão pode ser definida basicamente como a degradação de um metal (ou liga) a partir do contato de sua superfície com o meio no qual este esteja inserido. Neste sentido, muitos problemas relacionados à corrosão das armaduras têm influência das características do concreto, cuja função é oferecer uma dupla proteção ao aço. Primeiramente, tem-se a proteção física com a separação do aço e do meio externo pela camada de cobrimento, ao passo que a proteção química decorre do elevado $\mathrm{pH}$ da solução presente nos poros do concreto e da formação de uma película passivadora que envolve o aço no interior do concreto (FIGUEIREDO et al., 2012).

Dentre os fatores que influenciam o processo de corrosão do aço imerso no concreto estão: o coeficiente de difusão do concreto, a relação água/cimento, a espessura de cobrimento, a presença e a quantidade de adições, a umidade relativa, o pH do concreto e a temperatura de exposição (ANDRADE, 2001; GU et al., 1998). A Figura 1 ilustra a ação da corrosão. Nela pode-se perceber a ocorrência concomitante de duas situações, isto é, fissuras da camada de cobrimento do concreto e manchas corrosivas (CASCUDO, 1997). Para situações em que o concreto esteja com elevado grau de umidade nos poros, os óxidos são gerados a uma velocidade constante e podem migrar através da rede de poros, aparecendo na superfície sob a forma de manchas marrom-avermelhadas, mas não apresentando fissuras, Figura 1(a).

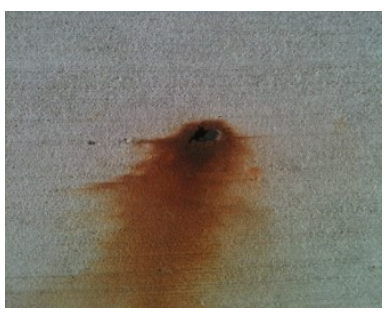

(a)

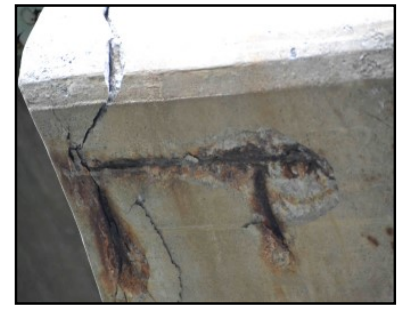

(b)

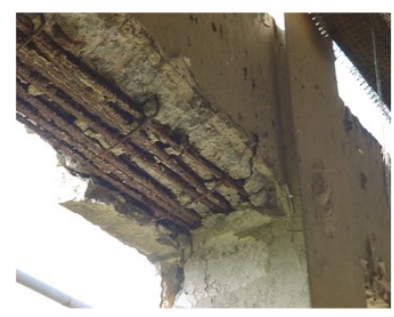

(c)

Figura 1 - Efeitos da corrosão na camada de cobrimento de concreto: (a) manchas (WILSON, 2018); (b) fissuras e rachaduras (SILVA, 2020); (c) destacamento total (CARVALHO, 2014)

A Figura 2 apresenta a perda de seção na região anódica pela dissolução do ferro. Tal perda provoca redução de aderência entre aço e concreto, redução da capacidade estrutural da peça e surgimento de manchas externas. Tensões internas expansivas decorrentes da corrosão (situados na região anódica) provocam a degradação da ferragem e o destacamento da camada de cobrimento, tal como apresenta as imagens da Figura 1 (b-c), A corrosão pode provocar fissuras paralelas às armaduras; fragmentação e destacamento do cobrimento e lascamento do concreto. Tal externalização do processo corrosivo normalmente determina que a armadura já está comprometida (ANDRADE, 2001; LOPES et al., 2018).

Outra importante característica é o fato de que, em sua maioria, as inspeções para avaliar o grau de corrosão são realizadas visualmente por especialistas humanos. Estas demandam altos níveis de 
experiência e são decisivas para uma correta avaliação do estado de degradação da estrutura (ABNT NBR $6118,2004)$.

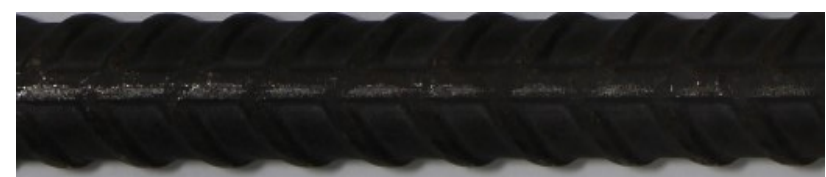

( a )

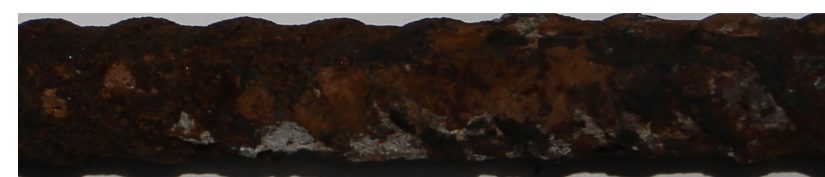

( b )

Figura 2 - Barra de aço: (a) original; (b) perda de seção devido à degradação. Obs.: para melhorar a qualidade das ilustrações, as imagens não mantêm a razão altura e largura originais.

As análises de materiais de construção por imagens têm sido objeto de diversos estudos recentes. Meira et al. (2020) analisaram a porosidade do concreto em mais de 2 mil microtomografias. Dias et al. (2012) correlacionaram imagens à deformação e fissuração de estruturas de concreto armado. Rocha et al. (2019) detectaram a corrosão de armaduras por meio de imagens termogravimétricas.

Diante do exposto, conclui-se pela necessidade de estudos pertinentes ao melhor entendimento dos processos de degradação de estruturas devido à corrosão com vistas a edificar estruturas mais seguras e duráveis. Este artigo busca auxiliar o processo de inspeção por meio da aplicação de sistemas baseados em visão computacional para classificar graus de degradação em barras de aço e reduzir a influência de fatores humanos nos resultados das inspeções. Tais sistemas contemplam, em geral, as seguintes etapas: aquisição, pré-processamento, segmentação, extração de características e classificação. Cada etapa constitui um diferente contexto, possui diferentes níveis de complexidade e envolve conhecimentos específicos, além dos inerentes ao domínio da aplicação. Já as influências compreendem a subjetividade do especialista humano e as características do processo (repetitivo, monótono e demorado), com a sobrecarrega do profissional, possíveis distrações e baixas taxas de acerto (CONNERS et al., 1997; PHAM et al., 1997; RADOVAN et al., 2001).

\section{Materiais e métodos}

A aquisição neste trabalho está relacionada à construção da base de imagens e envolveu 24 amostras, seis delas formaram o grupo de controle (referências isentas de corrosão) e outros três grupos de seis barras submetidos a processos de aceleração de corrosão por períodos de 24, 48 e 72 horas (Figura 3).

Para a construção da base de imagens foram utilizadas barras de aço com $12,5 \mathrm{~mm}$ de diâmetro. Cada amostra é caracterizada por um comprimento com aproximadamente $25 \mathrm{~cm}$, sendo que todas foram obtidas de barras pertencentes a um mesmo lote de fabricação, Figura 3(a). Suas extremidades tiveram 10 $\mathrm{cm}$ cobertos por fita isolante para garantir que a corrosão ocorresse apenas na parte central da amostra. Um fio elétrico foi conectado a cada barra com o objetivo de permitir o ensaio de aceleração da corrosão.

A amostra foi inserida em um cano de PVC com diâmetro nominal de $50 \mathrm{~mm}$ e $25 \mathrm{~cm}$ de comprimento. Este cano de PVC foi preenchido com concreto cujo traço tinha a proporção $1: 1,41: 2,01: 0,49$ (Cimento : Areia : Brita: Água). Para o traço de concreto foi utilizado cimento do tipo Portland CPII, areia média, brita zero (dimensão máxima característica igual a $9,5 \mathrm{~mm}$ ) e água potável disponibilizada pela companhia de saneamento. A cobertura se manteve homogênea em termos de espessura, aproximadamente $2 \mathrm{~cm}$, o que caracteriza medida semelhante ao contexto real das estruturas nas edificações, Figura 3(b). As amostras permaneceram sob cura por 28 dias para o processo de hidratação do cimento, Figura 3(c).

Seguiu-se com o processo de aceleração de corrosão das amostras com os corpos de concreto parcialmente imersos em um tanque de água com 3,5\% (concentração próxima a água do mar) de cloreto de sódio ( $\mathrm{NaCl}$ ) e submetidos ao ensaio de aceleração da corrosão através da condução de eletricidade. Os grupos de corpos de concreto foram mantidos por três diferentes períodos de tempo no tanque (24, 48 e 72 horas), Figura 3(d-f), gerando diferentes graus de corrosão nas armaduras, Figura 3(g-i). 
Cessado o processo de aceleração de corrosão, os corpos de concreto, bem como as barras internas depois de extraídas e limpas, foram submetidos a uma coleta de imagens para compor a base de imagens. Para cada amostra de barra, foram capturadas cinco imagens abrangendo a diferentes perspectivas da superfície dos corpos de concreto e também da barra depois de extraída, num total de 120 imagens para cada caso. No presente estudo foram consideradas as imagens relativas às barras, Figura 3(g-i).

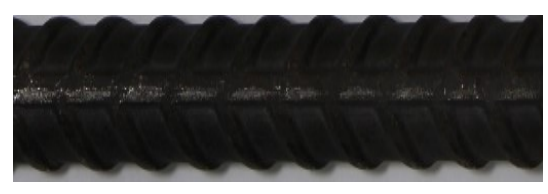

(a)

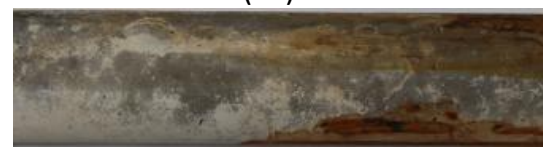

(d)

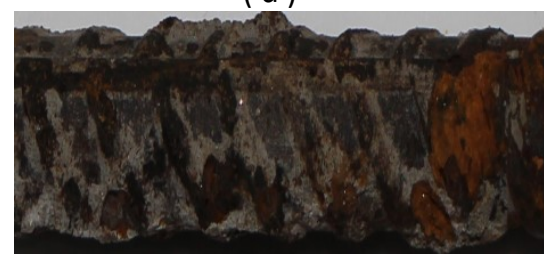

(g)

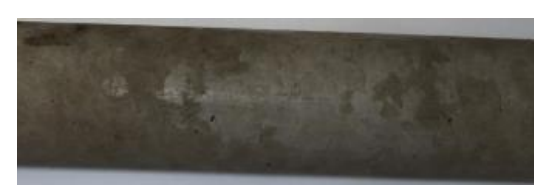

(b)

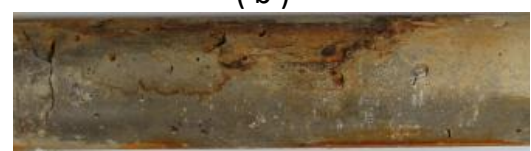

(e)

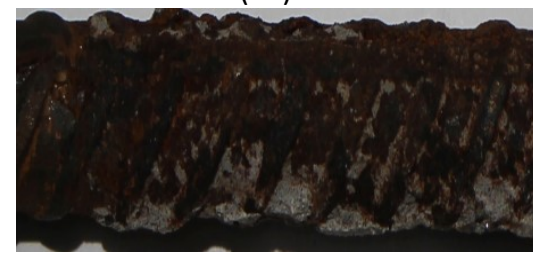

(h)

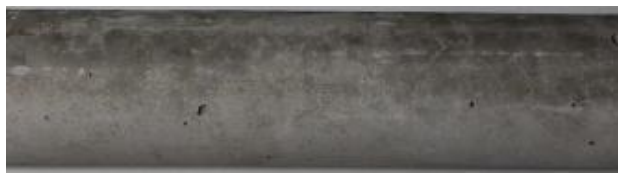

(c)

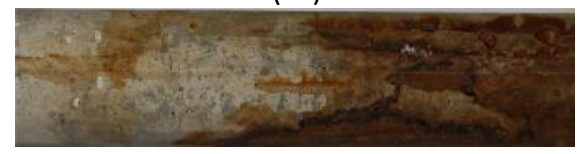

(f)

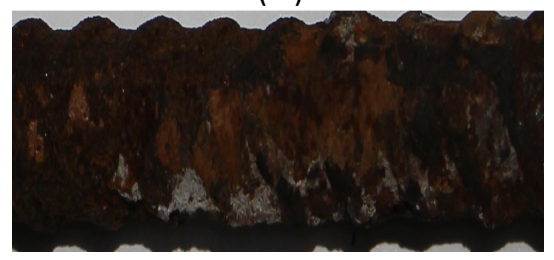

(i)

Figura 3 - Processo de construção da base de imagens: ( a ) barra original; ( b ) corpo de concreto envolvendo a barra original; ( c ) corpo de concreto envolvendo a barra após 28 dias de cura do concreto; ( d-f ) corpos de concreto envolvendo a barra após o processo de aceleração (respectivamente 24, 48 e 72 horas); (g-i) barras retiradas dos corpos de concreto após o processo de aceleração (respectivamente 24, 48 e 72 horas). Obs.: para melhorar a qualidade das ilustrações, as imagens não mantêm a razão altura e largura originais.

A Figura 4 ilustra os resultados das etapas de pré-processamento e segmentação, sendo que a primeira aplica operações para realçar as características importantes no processo de diferenciação das classes existentes no problema. Nesta etapa utilizou-se principalmente da biblioteca OpenCV para converter todas as imagens da base para escala de cinza, conforme ilustração das imagens da Figura 4(a-b).

A segmentação, par de imagens da Figura 4(c-d), busca separar apenas a região de interesse da imagem original, tendo-se buscado por imagens que estivessem todas formadas pela área de interesse, imagens da Figura 4(c-d). Foram utilizados a técnica de limiarização para destacar os objetos na imagem, o algoritmo de deteç̧ão de bordas Canny (BUENO, 2020) para ressaltar os contornos das bordas destes objetos e a função findContours da biblioteca OpenCV para identificar os pontos que pertenciam a tais contornos. Esta função retorna os pontos que formam os contornos de todos os possíveis objetos presentes na imagem, os quais são caracterizados por qualquer região com contraste em relação a seu entorno. Identificados os possíveis objetos, os pontos pertencentes ao maior contorno com a definição da barra de aço foram selecionados. Ao final, recortou-se e salvou-se a área de interesse em uma nova imagem, imagens da Figura 4(c-d).

A etapa de extração de características busca representar a imagem por meio de um vetor numérico de medidas. Nesta etapa foram utilizados os descritores LBP (Local Binary Pattern) (MÄEMPÄ et al., 2000; OJALA et al., 1996); SURF (Speed-Up Robust Feature) (BAY et al., 2006, 2008) e LPQ (Local Phase Quantization) (OJANSIVU et al., 2008). Detalhes quanto aos descritores e também à abordagem adotada para sua extração podem ser obtidos em Martins (2014).

$\mathrm{Na}$ etapa de classificação foi utilizado o algoritmo Máquina de Vetores de Suporte (Support Vector Machine - SVM), por meio da implementação LibSVM 3.2 (CHANG et al., 2011). Os melhores resultados foram obtidos com a função de base radial Gaussiana e uma busca gulosa para encontrar os melhores parâmetros 
C e $\gamma$, bem como otimizar as taxas finais de reconhecimento. A normalização dos dados considerou a escala linear de cada atributo dos vetores de características no intervalo $[-1,+1]$.

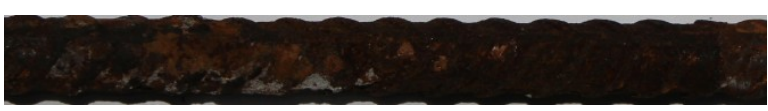

( a )

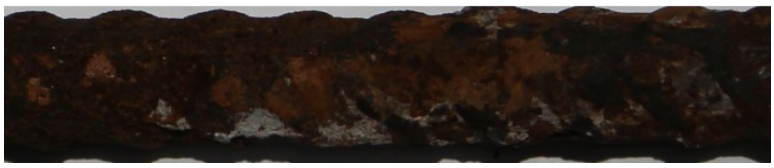

(c)

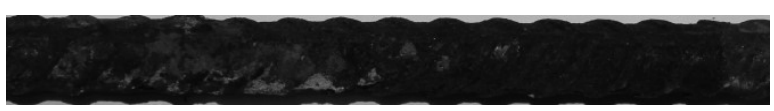

(b)

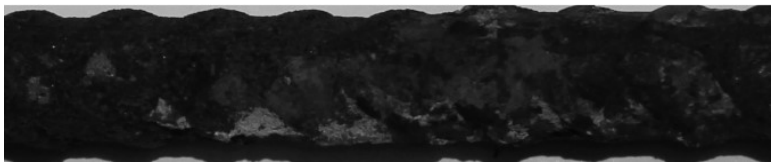

(d)

Figura 4 - Processo de reconhecimento de padrões: ( a ) barra após o processo de aceleração; ( b ) pré-processamento com conversão para níveis de cinza; ( c-d ) segmentação. Obs.: para melhorar a qualidade das ilustrações, as imagens não mantêm a razão altura e largura originais.

Para a definição dos conjuntos de treinamento e teste, dividiu-se a base de imagens em três partes (folds), as quais mantinham a representatividade das classes pertencentes ao problema em questão. Foram realizadas três execuções, sendo que em cada execução uma parte era usada como conjunto de teste e as outras duas eram utilizadas como conjunto de treinamento. Os resultados finais são expressos pela média e desvio padrão das taxas de classificações corretas das três execuções.

Neste trabalho foi considerada a métrica acurácia, caracterizada pela razão entre as predições corretas totais e o número de amostras. A métrica foi calculada para cada fold. A partir destes valores individuais foram calculadas a acurácia média e o desvio padrão ( $\mathrm{dp}$ ), sendo que este último nos permite avaliar o quão representativos são os conjuntos de treinamento e teste utilizados à medida que tende a zero.

\section{Resultados e discussão}

O Quadros 1 e 2, bem como a Figura 5, apresentam os resultados obtidos com três execuções utilizando variações dos descritores LBP, SURF e LPQ. Do Quadro 1 tem-se que o melhor resultado dentre as variações do descritor LBP foi obtido por LBP riuz e sua invariância à rotação das imagens, a qual alcançou 73,3\% (dp = 6,2). O Quadro 2 apresenta taxas de reconhecimento menores, com acertos de até $70,8 \%(\mathrm{dp}=1,2)$ para a variante do SURF com 64 atributos e momento estatístico variância.

Quadro 1 - Taxas de reconhecimento alcançadas com o descritor LBP

\begin{tabular}{|c|c|c|c|c|}
\hline Versão do LBP & $\mathbf{P}$ & $\mathbf{R}$ & Acurácia (\%) & $\mathbf{d p}$ \\
\hline \multirow{3}{*}{ riu2 } & 8 & 1 & 69,2 & 4,7 \\
\cline { 2 - 5 } & 16 & 2 & $\mathbf{7 3 , 3}$ & 6,2 \\
\cline { 2 - 5 } & 24 & 3 & 72,5 & 8,2 \\
\hline \multirow{3}{*}{ u2 } & 8 & 1 & 35,8 & 20,9 \\
\cline { 2 - 5 } & 16 & 2 & $\mathbf{6 7 , 5}$ & 12,4 \\
\cline { 2 - 5 } & 24 & 3 & 52,5 & 22,5 \\
\hline
\end{tabular}

As taxas de reconhecimento dos Quadros 1 e 2, com vantagens para as versões com menos elementos nos vetores de características, podem ser explicadas pela forma como os descritores são computados. A menor quantidade de elementos nos vetores de características destas variantes decorre da concentração ou 
acúmulo de conjuntos de características em um único elemento, o que gera invariância à rotação. Neste ponto, destaca-se que a acomodação das britas no corpo de concreto ocorre de forma aleatória, não podendo ser controlada, e que tal fato é representado também nas imagens capturadas.

A Figura 5 apresenta os resultados alcançados com a aplicação do descritor $L P Q$ e a variação de seus parâmetros relacionados ao tamanho da janela (eixo X) e tipo do filtro utilizado (linhas). As melhores taxas de reconhecimento foram obtidas pela combinação do filtro baseado em Janelas Gaussiana com dimensões $11 \times 11$, alcançando $75,6 \%(d p=6,3)$, as quais também constituem os melhores resultados gerais.

Quadro 2 - Taxas de reconhecimento alcançadas com o descritor SURF

\begin{tabular}{|c|c|c|c|}
\hline Versão do SURF & Medida & Acurácia (\%) & dp \\
\hline \multirow{4}{*}{64} & \# Pontos & 52,5 & 2,0 \\
\cline { 2 - 4 } & Média & 61,7 & 2,4 \\
\cline { 2 - 4 } & Variância & $\mathbf{7 0 , 8}$ & 1,2 \\
\cline { 2 - 4 } & Obliquidade & 47,5 & 15,9 \\
\cline { 2 - 4 } & Curtose & 46,7 & 9,4 \\
\hline \multirow{4}{*}{128} & \# Pontos & 52,5 & 2,0 \\
\cline { 2 - 4 } & Média & 58,3 & 7,7 \\
\cline { 2 - 4 } & Variância & $\mathbf{6 1 , 7}$ & 6,6 \\
\cline { 2 - 4 } & Obliquidade & 49,2 & 17,8 \\
\cline { 2 - 4 } & Curtose & 34,2 & 13,0 \\
\hline
\end{tabular}

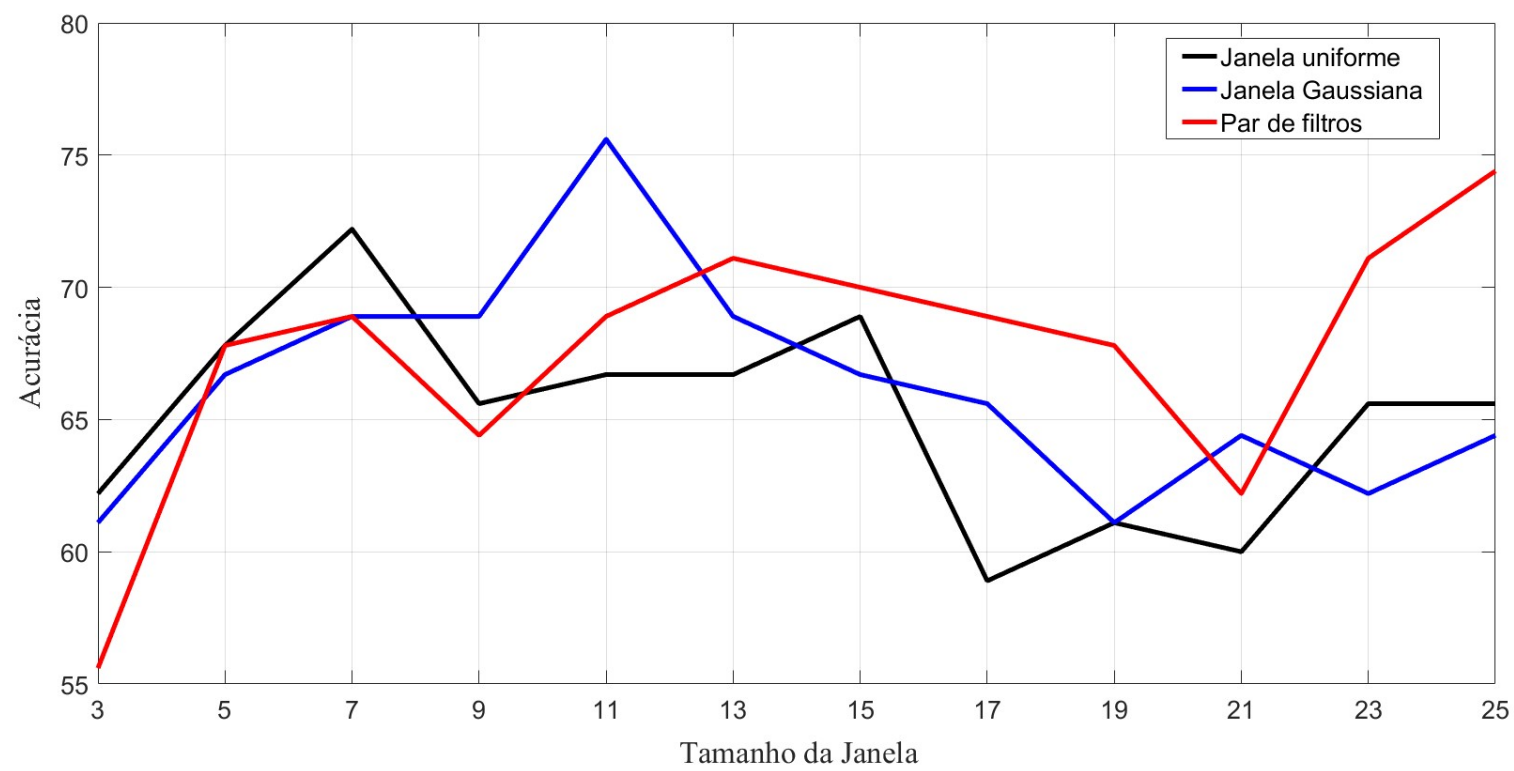

Figura 5 - Variação das taxas de reconhecimento alcançadas com o descritor LPQ

As taxas de reconhecimento se mostram bastante interessantes diante das dificuldades que um especialista humano enfrenta ao tentar identificar visualmente o grau de degradação nas barras, Figura 3(g-i). 


\section{Conclusões}

Este trabalho abordou o problema de classificação do grau de degradação de barras de aço por meio de imagens. Os melhores resultados foram alcançados pelo descritor $L P Q$, com acurácia de $75,6 \%(d p=6,3$ ), mediante combinação do filtro baseado em Janelas Gaussiana com dimensões 11x11. Diante da complexidade do problema apresentado, os resultados obtidos validaram a proposta de se empregar sistemas computacionais que trabalhem com visão computacional para auxiliar ou substituir especialistas humanos no processo de identificação de concretos.

A partir destes resultados preliminares, vislumbra-se a continuidade dos trabalhos com a avaliação de novas alternativas para descritores, classificadores, estratégias de validação e também a seleção e combinação de modelos, com o intuito de melhorar ainda mais os resultados. Possíveis alternativas foram apresentadas por Martins et al. (2012), Martins (2014), Martins et al. (2015) e Spanhol (2018).

\section{Referências Bibliográficas}

ASSOCIAÇÃO BRASILEIRA DE NORMAS TÉCNICAS - ABNT NBR 6118 (2004). ABNT NBR 6118: Projeto de estruturas de concreto - Procedimento. Rio de Janeiro, 2004.

ANDRADE, J. J. de O. (2001). Contribuição à previsão da vida útil das estruturas de concreto armado atacadas pela corrosão de armaduras: iniciação por cloretos. Tese de Doutoramento. Programa de PósGraduação em Engenharia Civil. Universidade Federal do Rio Grande do Sul.

BAY, H.; TUYTELAARS, T.; e VAN GOOL, L. (2006). SURF: Speeded up robust features. ECCV, 404-417.

BAY, H.; ESS, A.; TUYTELAARS, T.; e VAN GOOL, L. (2008). Speeded-up robust features (SURF). Compute Vision. Image Understanding, 110 (3), 346-359.

BUENO, M. L. Deteç̧ão de Bordas através de Algoritmo Canny. Disponível em: http://www.inf.ufsc.br/ aldo.vw/visao/2000/Bordas/index.htm. Acesso em: 20 jan. 2020.

CARVALHO, R. R. Patologias em concreto armado, 30 de mai. de 2014. Disponível em: https://www.rodrigorcarvalho.com.br/patologias-em-concreto-armado/. Acesso em: 20 de jun. de 2020.

CASCUDO, O. (1997). O Controle da Corrosão de Armaduras em Concreto. Goiânia: PINI e UFG.

CHANG, C.-C.; LIN, C.-J. (2011). LIBSVM: a library for support vector machines. ACM Transactions on Intelligent Systems and Technology, 2(3), 1-27. Disponível em: http://www.csie.ntu.edu.tw/ cjlin/libsvm/. Acesso em: 20 de jun. de 2020.

CONNERS, R. W.; KLINE, D. E.; ARAMAN, P. A.; DRAYER, T. H. (1997). Machine vision technology for the forest products industry. Computer, 30 (7), 43-48.

CUNHA, M.; HELENE, P.; LOURENÇO, M. (2013). Corrosão em estruturas de concreto armado: teoria, controle e métodos de análise. Elsevier Brasil.

DIAS, R.P.; SOUZA, J. L. A. O.; ALMEIDA L. C. (2012). Aplicação da técnica de correlação de imagens digitais à análise experimental de estruturas de concreto armado. Cong. Interno de Inic. Científica da UNICAMP. Campinas, outubro de 2012. Disponível em: https://www.prp.unicamp.br/pibic/congressos/ xxcongresso/paineis/082742.pdf. Acesso em: 09 fev. 2021.

FIGUEIREDO, E. P.; MEIRA, G. (2012). Corrosão das armaduras das estructuras de concreto. Boletín Téc. 6. Asociación Latino americana de Control de Calidad, Patología y Recuperación de la Construcción. Mexico.

GU, P.; BEAUDOIN, J. J. (1998). Obtaining effective half-cell potential measurements in reinforced concrete structures. Construction Technology Update No. 18. National Research Council of Canada, Institute for Research in Construction. Ottawa.

LOPES, F. L. C.; MONTEIRO, M. D. S.; MELO, H. C. S.; SIQUEIRA, L. M.; LOPES, F. L. G. (2018). Análise quantitativa da perda de massa por oxidação em barras de aço carbono CA-50: uma proposta metodológica para a contextualização do estudo da corrosão em cursos de engenharia civil/IFS. Simpósio de Engenharia de Produção de Sergipe, 10, São Cristóvão, SE, 440-452. 
MÄENPÄÄ, T.; OJALA, T. PIETIKÄINEN, M.; SORIANO, M. (2000). Robust texture classification by subsets of local binary patterns. 15th International Conference on Pattern Recognition, 947-950.

MARTINS, J.G. (2014). Identificação de Espécies Florestais utilizando Seleção Dinâmica de Classificadores no Espaço de Dissimilaridade. Tese de Doutoramento. Programa de Pós-Graduação em Informática. UFPR, Curitiba-PR.

MARTINS, J.G.; OLIVEIRA, L.E.S.; SABOURIN, R. (2012). Combining textural descriptors for forest species recognition. In IECON 2012 - 38th Annual Conference on IEEE Industrial Electronics Society, 1483-1488.

MARTINS, J.G.; OLIVEIRA, L.E.S.; BRITTO, A. S.; SABOURIN, R. (2015). Forest species recognition based on dynamic classifier selection and dissimilarity feature vector representation. Machine Vision and Applications, 26 (2), 279-293.

MEHTA, P.K.; MONTEIRO, P. (2008). Concreto: Microestrutura, Propriedades e Materiais. 3.ed. São Paulo: IBRACON.

MEIRA, S. A.; SACRAMENTO, L. A.; LIMA, M.P.; PESSOA, J. R. C.; NASCIMENTO, F. L. A.; ASSIS, J. T. (2020). Análise da porosidade de concreto por processamento de imagem: uma visão da sensibilidade do threshold na binarização. Brazilian Journal of Development, 6(3), 16449-16459.s

OJALA, T.; PIETIKÄINEN, M. ; HARWOOD, D. (1996). A comparative study of texture measures with classification based on featured distributions. Pattern Recognition, 29(1), 51-59.

OJANSIVU, V. ; HEIKKILÄ, J. (2008). Blur insensitive texture classification using local phase quantization. Proceedings of the 3rd Intern. Conference on Image and Signal Processing, Berlin, Heidelberg, 236-243.

PHAM, D. T.; ALCOCK, R. J. (1997). Automated visual inspection of birch wood boards. IEE Colloquium on Artificial Intelligence in Manufacturing, 1-4.

PREFEITURA de SP interdita ponte que leva à Dutra pela Marginal Tietê. G1 SP, São Paulo - SP, 23 de jan. de 2019. Disponível em: https://g1.globo.com/sp/sao-paulo/noticia/2019/01/23/prefeitura-de-spinterdita-ponte-que-leva-a-dutra-pela-marginal-tiete.ghtml. Acesso em: 20 de jun. de 2019.

RADOVAN, S.; GEORGE, P.; PANAGIOTIS, M.; MANOS, G.; ROBERT, A.; IGOR, D. (2001). An approach for automated inspection of wood boards. International Conference on Image Processing, 1, 798-801.

ROCHA, J. H. A.; PÓVOAS, Y.V. (2019). Detection of corrosion in reinforced concrete with infrared thermography and ultrasound. Ambiente Construído, 19(3), 53-68.

SILVA, M. V. F., PEREIRA, M. C., CODARO, E. N. (2015). Corrosão do aço-carbono: uma abordagem do cotidiano no ensino de química. Química Nova, 38 (2), 293-296. São Paulo.

SILVA, M. N. Como evitar o ataque por cloretos em edificações próximas ao mar, 24 de abr. de 2020. Disponível em: https://www.sienge.com.br/blog/ataque-por-cloretos/. Acesso em: 20 de jun. de 2020.

SPANHOL, F. A. (2018). Automatic Breast Cancer Classification From Histopathological Images: A Hybrid Approach. Tese de Doutoramento. Programa de Pós-Graduação em Informática. UFPR, Curitiba-PR.

VIADUTO da Galeria dos Estados desaba e abre cratera no Eixão Sul. Correio Braziliense, Brasília - DF, 06 de fev. de 2018. Disponível em: https://www.correiobraziliense.com.br/app/noticia/cidades/2018/02/06/

interna_cidadesdf,658118/parte-do-viaduto-da-galeria-dos-estados-desaba-no-centro-de-brasilia.shtml. Acesso em: 20 de jun. de 2019.

WILSON, A. Three Most Common Concrete Stains, 04 de jun. de 2018. Disponível em: https://www.sienge.com.br/blog/ataque-por-cloretos/. Acesso em: 20 de jun. de 2020. 